\title{
Biology of Maize Kernel Infection by Fusarium verticillioides
}

\author{
Keith E. Duncan and Richard J. Howard \\ DuPont Agricultural Biotechnology, Experimental Station, 200 Powder Mill Road, Wilmington, DE 19880-0353, U.S.A.
}

Submitted 28 July 2009. Accepted 19 September 2009.

\begin{abstract}
Fusarium kernel rot disease starburst symptomatology was characterized fully for the first time. Two maize lines were hand pollinated and inoculated, using a fluorescent protein-expressing transformant of the fungal pathogen Fusarium verticillioides, by introduction of a conidial suspension through the silk channel of intact ears. Microscopy was used to identify the infection court and document initial stages of kernel colonization and subsequent manifestation of macroscopic symptoms. The fungus entered kernels of susceptible line AD38 via an open stylar canal and spread extracellularly and over the kernel through the nucellus region, sporadically entering pericarp and filling the long thick-walled mesocarp cells. Hyphae spread within pericarp from cell to cell via pits, colonizing files of host cells by growing both up and down the kernel in a radial pattern that preceded macroscopic symptom development. The starburst symptom developed subsequently, and mirrored colonization exactly, when there was extensive dissolution of the thick walls of pericarp cells. Line HT1 exhibited a closed stylar canal phenotype and was not susceptible-except when the pericarp surface was breached mechanically. We hypothesize the passive movement of conidia along the surface of silks, perhaps via capillarity, as a possible mechanism for pathogen access to the infection court.
\end{abstract}

The fungus Fusarium verticillioides (Sacc.) Nirenberg (Nirenberg and O'Donnell 1998), as a pathogen of Zea mays, is a concern primarily because it produces secondary metabolites toxic to animals and man (Munkvold 2003; Bacon et al. 2008). Furthermore, the fact that it infects a plant representing a significant component in the human food supply chain-often assuming the stealthy habits of an endophyte-underscores an obvious need for understanding the biology of host-pathogen interaction as thoroughly as possible.

F. verticillioides is thought to commonly exist systemically and asymptomatically in most field corn (in roots, stalk tissues, and kernels) and to be passed from parent to progeny by seedborne infection (Wilke et al. 2007). In addition, kernel rot is often associated with damaged host tissues; for example, from the feeding of insects (Maiorano et al. 2009; Munkvold and Hellmich 2000; Sobek and Munkvold 1999; Warfield and Davis 1996) or mechanical harvesting (Munkvold 2003). The mechanisms by which otherwise undamaged and uninfected plants are invaded has remained unclear but may be significant (Maiorano et al. 2009).

Corresponding author: Richard J Howard:

E-mail: richard.j.howard@cgr.dupont.com

*The Spotlight logo represents articles that, in the opinion of the senior editor and editor-in-chief, are of special interest to a broad readership.
The biology leading to the classic starburst symptom consisting of streaks on the pericarp radiating from the silk scar region of kernels is poorly understood. The white color of these streaks has been reported to be caused by a loss of transparency due to the disintegration of pericarp cells (Koehler 1942), presumably as a direct result of fungal action on those cells. However, since the early work of Koehler, there have been conflicting reports and speculation about how this and closely related pathogens gain access to kernels and, subsequently, how these fungi enter intact kernels, even whether streak development is a direct result of pathogenesis.

Kernel infection can result from inoculation of exposed silks using a conidial suspension (Jones et al. 1980; Headrick and Pataky 1991; Reid et al. 1992; Munkvold et al. 1997), while wounds to the husk or pericarp have been cited as sufficient or necessary (Warfield and Davis 1996; Yates and Sparks 2008). A strong correlation has been reported between levels of fumonisin contamination of kernels and factors that appear to affect infection via silks, i.e., duration of silking and silk wetness (Maiorano et al. 2009). An avenue of direct kernel ingress has not been identified. To our knowledge, there are no reports documenting the kernel infection process following silk inoculation in the absence of wounds (i.e., reports that determined the precise infection court, or if one exists). In a detailed field study, Hesseltine and Bothast (1977) analyzed the progression of organisms into various ear tissues over a 15-week period, and carefully noted whether injuries were apparent. From their observations, they concluded that "shortly after pollination the silks became dead tissue, serving as a substrate... and acting as a highway to the kernels" for various organisms. Headrick and associates (1990) isolated the pathogen from silk tissue sampled after inoculation and reported evidence for apparent movement from exposed regions to regions under the husk. Valdivia and associates (2006), Stewart and associates (2002), and Reid and associates (2002) reported field results of reduced fungal ear rot correlated with accelerated silk senescence and longer intervals of time between silk emergence and silk channel inoculations but how these factors were related remains unknown. Working with the ear rot pathogen F. graminearum, Miller and associates (2007) provided evidence of fungal hyphae growing on the surface of silks as well as internally and, thus, entering the intact ear via the silk channel. Beginning with Koehler (1942), many others have also concluded a priori that the fungus gains access to kernels through the silk channel (Wolf et al. 1952; Vigier et al. 1997; Dowd 1998; Stewart et al. 2002; Rossi and Scandolara 2009)—bypassing the protective husks-but no direct and convincing observations of a kernel infection court have been published.

Here, we report observations of the disease process using a fluorescent protein-expressing transformant of the pathogen and, 
for the first time, document the stylar canal as an infection court that allows the fungus to enter unwounded kernels.

\section{RESULTS}

\section{Growth of the fungus on silk tissue.}

Initially, we hypothesized that $F$. verticillioides gained access to the corn kernel by growing along or within the stigmatic structures (silks), possibly in association with the pollen tube, and used the attachment point of the silk to invade the kernel. To test this possibility, a conidial suspension of ZsGreen-expressing $F$. verticillioides was sprayed onto exposed pollinated and unpollinated silks of both the susceptible line AD38 and the resistant line HT1, and the pattern and extent of fungal growth was evaluated over time, from days to weeks. Using fluorescence microscopy and cryo scanning electron microscopy (cryoSEM), we observed extensive fungal growth and sporulation on pollinated silks; specifically, on and around pollen grains, with copious conidia produced within $72 \mathrm{~h}$ postinoculation (Fig. 1). However, this growth and sporulation was evident only on the exposed pollinated silks, with a significant reduction in growth on silks that were covered by the husk and nonsenescent (discussed below). Similarly, unpollinated nonsenescent silks supported only minimal fungal growth both above and below the husk line.

The two corn lines used in this study differed in husk coverage and silk channel length (the distance from the tip of the developing ear to the top edge of the husk). Resistant line HT1 was wrapped more tightly and with more layers than susceptible line AD38. As a result, the silk channel in HT1 was longer

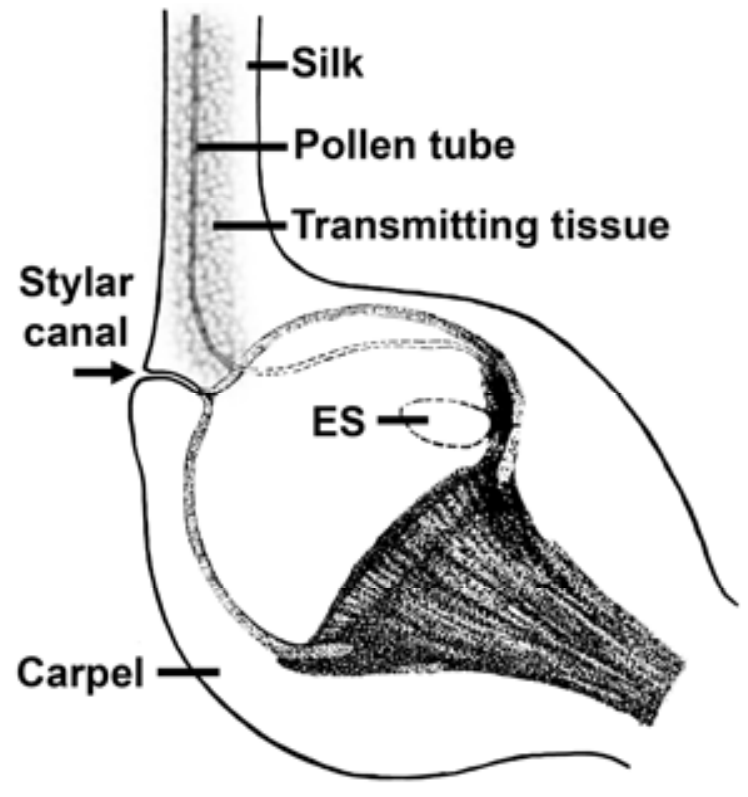

Fig. 2. Diagram of a cross-section through a developing corn kernel at pollination showing the orientation of the intact silk and the convergence of the carpels that forms the stylar canal. The path of the pollen tube is shown as it grows through the transmitting tissue of the silk (indicated by shaded area; see Heslop-Harrison et al. 1984), across the face of the nucellus (where the pollen tube is indicated by dotted line), and into the embryo sac (ES). Redrawn from Kiesselbach 1949 and used with permission of the copyright holder, Cold Spring Harbor Laboratory Press.
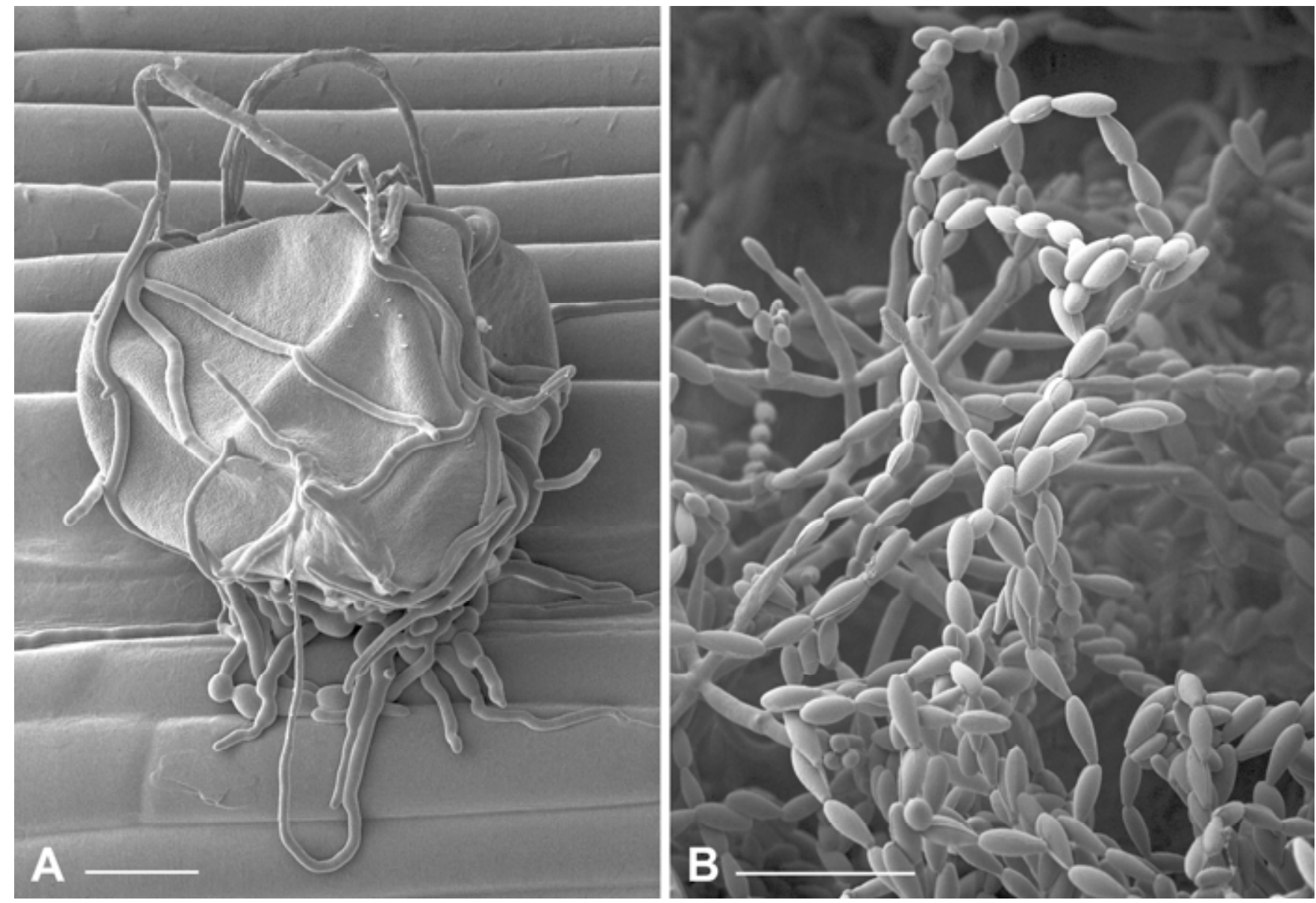

Fig. 1. Cryo scanning electron microscope images taken from the surface of exposed maize silk (line AD38) 48 and $96 \mathrm{~h}$ after hand pollination and 24 and 72 $\mathrm{h}$ after spray inoculation. A, Most fungal growth was found in association with pollen grains; note numerous hyphae on the surface of the single pollen grain depicted. B, Copious production of Fusarium verticillioides conidia. Bars represent $20 \mu \mathrm{m}$. 
and the silks were more tightly pressed together than in AD38. Fungal growth on the surface of pollinated nonsenescent HT1 silks was halted within $1 \mathrm{~cm}$ of the husk line, whereas growth along nonsenescent AD38 silks was typically evident 2 to $3 \mathrm{~cm}$ below the husk line before halting. In all cases, fungal growth was restricted to the surface of the silks and we found no evidence that $F$. verticillioides routinely invaded intact corn kernels by growing along or within silks to gain access.

\section{Kernel infection.}

Confocal laser scanning microscopy (LSM) and cryoSEM observations of areas inside the husks, after injection inoculation, demonstrated that growth of the fungus on surfaces was limited, found primarily on floral parts surrounding the developing kernel, with little growth evident on most of the surface of the kernel. However, we consistently found growth of $F$. verticillioides at the base of the silk scar on AD38 but not on HT1, in the region of the stylar canal (Fig. 2). The stylar canal was conspicuously open and at a level somewhat raised and separated from the silk scar in AD38, but appeared as a closed seam and more integrated with the silk scar in HT1. These differences in stylar canal architecture between the two corn lines were evident from early during kernel development (Fig. 3A and B) until maturity (Fig. 3C and D). Also in mature kernels, between the stylar canal and the silk scar, a trough was evident in AD38 versus a ridge in HT1 (Fig. 3D). Conidia and hyphae were observed in this trough as early as $24 \mathrm{~h}$ postinoculation (Fig. 4A). Pathogen structures were typically not found in this region of HT1 kernels. Fungal growth in the stylar canal was detected as early as 7 days postinoculation (dpi), in AD38 only, by observing whole intact kernels under a fluorescence stereomicroscope (Fig. 5A) with no corresponding macroscopic symptoms. As early as $14 \mathrm{dpi}$, we observed hyphae within (or under) the pericarp expanding outward from the stylar canal of AD38 kernels (Fig. 4B and C). There was a conspicuous shift in fungal morphology as it reached the inner end of the stylar canal and began to spread internally. Hyphae in the nucellus region were wider and bulbous, and more variable in morphology, compared with germ tubes or hyphae associated with the kernel surface (Fig. 4A, B, and C).

More detailed microscopy was afforded by observation of Vibratome sections. In AD38 kernels, we documented the presence of fluorescent hyphae within the entire length of the stylar canal and into the nucellar region (Fig. 5B and C). From there, it grew rapidly within the nucellus and outward-toward the kernel surface - into the long, radially arranged mesocarp cells. As these files of cells were colonized they comprised fluorescent radial streaks, centered on the stylar canal, matching the pattern of starburst symptoms that sometimes developed later (Fig. 5D). Frequently, there was not an obvious connection directly from the stylar canal to the outward radiating streaks or patches of fluorescence when intact kernels were viewed with the fluorescence dissecting microscope. We observed that fluorescence intensity was greatest in hyphal apices and diminished in older hyphae (Fig. 6A); therefore, under low magnification the connection from stylar canal to leading edge of fungal colonization
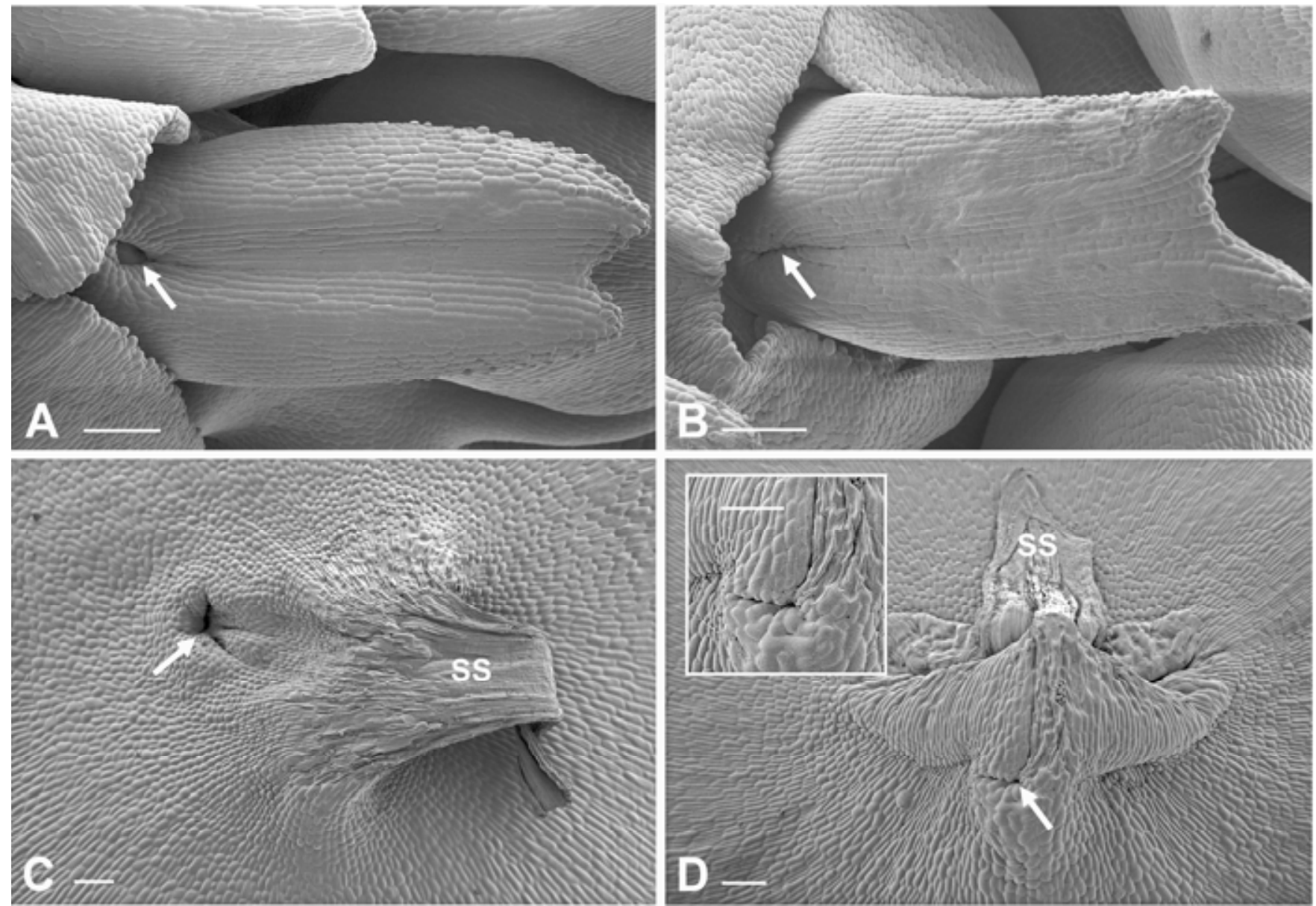

Fig. 3. Cryo scanning electron microscopy images of the stylar canal region of maize lines AD38 and HT1 kernels. Immature styles (silks) of A, AD38 and B, HT1 at 53 days post planting; top of kernels of $\mathbf{C}$, AD38 and D, HT1 at 28 days postpollination. Note that the stylar canal (arrows) is open in susceptible AD38 compared with closed in resistant HT1 (inset is enlarged area of same image). Also, note that, near the base of the silk scar (SS), is a trough at the mouth of the stylar canal in AD38 whereas, in HT1, a seam-like stylar canal is positioned on a raised ridge. Bars represent $100 \mu \mathrm{m}$. 
was not always visible. We were able to verify these connections under higher magnification.

Once the fungus entered the long cells of the mesocarp, it exhibited a lobed and highly branched, densely packed growth habit and filled the empty lumen of these cells (Fig. 6A and B). Multichannel LSM allowed tracing of the growth of the fungus laterally and vertically between long cells, apparently through numerous pit pairs in the thick secondary cell walls (Fig. 6C and D) (Wolf et al. 1952).

Growth of the fungus in the early stages of kernel infection via the stylar canal was robust and rapid, with the leading edges of hyphal expansion fluorescing brightly. In the later stages of this process, growth and expansion of the fungus slowed significantly, perhaps in response to the decreasing moisture content of the kernel. Transmission electron microscopy (TEM) observations of nonsymptomatic kernels showed no evidence for dissolution of invaded pericarp cell walls, even in cells that were packed densely with hyphae (Fig. 7A). However, in pericarp where macroscopic symptoms were evident, extensive dissolution of the host cell wall was observed and hyphae were no longer confined to the cell lumen but were found to have ramified through the thick secondary cell wall (Fig. 7B and C).

Despite a uniform exposure of each kernel to conidia of $F$. verticillioides, the extent to which kernels of a single ear were colonized varied from one or two fluorescent streaks with no macroscopic symptoms, to extensive fluorescent streaking coupled with macroscopic starburst pattern development, to com-

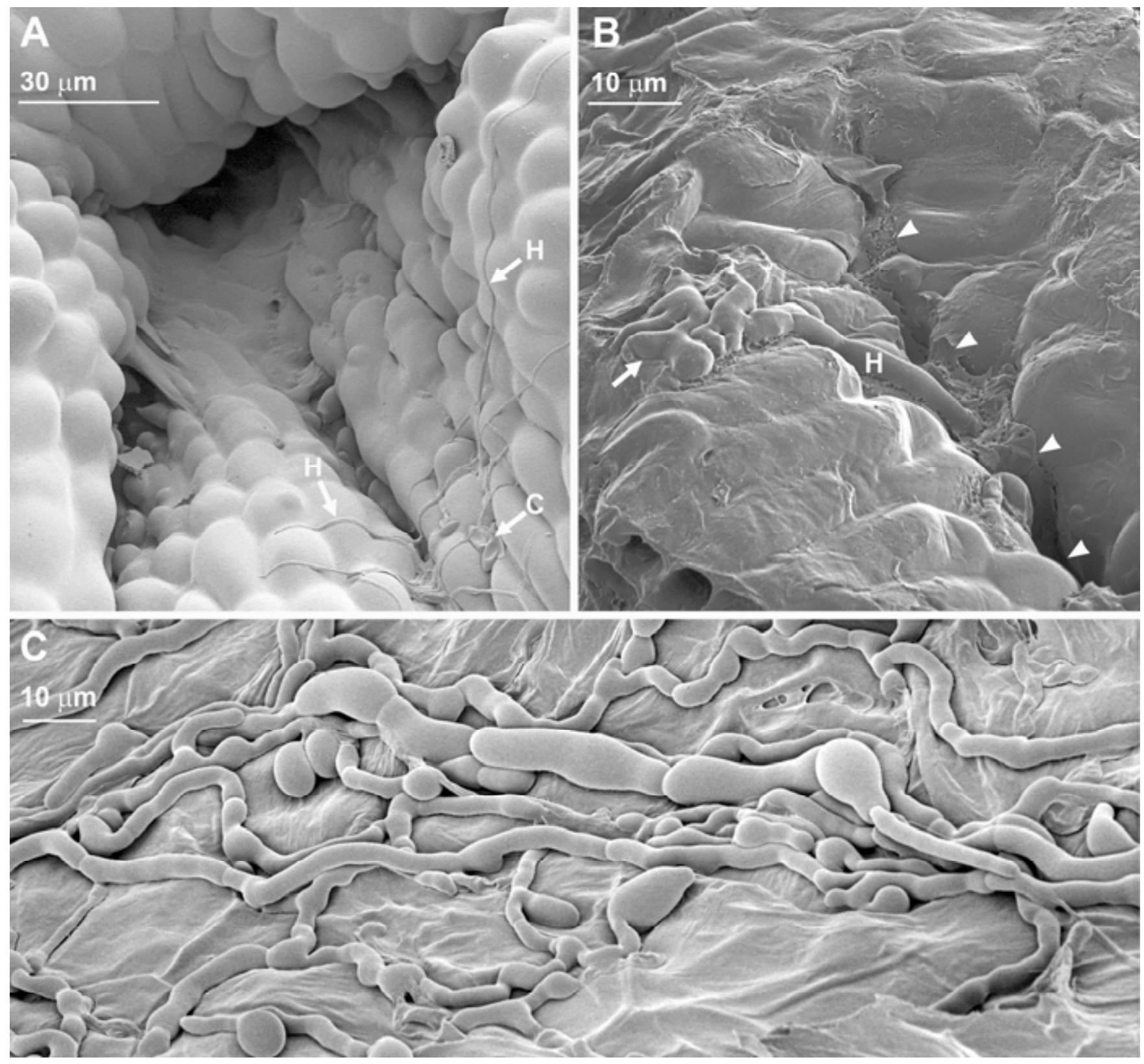

Fig. 4. Cryo scanning electron microscopy (cyroSEM) images of Fusarium verticillioides hyphae found A, on the pericarp surface and $\mathbf{B}$ and $\mathbf{C}$, underside at stylar canal regions of AD38 kernels 24 h, 16 days, and 17 days postinoculation, respectively. A, Hyphae $(\mathrm{H})$ and conidia $(\mathrm{C})$ in the trough in front of a stylar canal opening on the surface of the kernel. B, Underside of a pericarp, preselected by fluorescence dissecting microscope observation of ZsGreen-expressing hyphae within the stylar canal, peeled from the kernel, and mounted nucellus-side up for cryoSEM. A hypha (H) grew in a lobed and highly branched manner (arrow) after apparently emerging from the inside slit-shaped opening of the stylar canal (arrowheads). C, $F$. verticillioides growing underneath the pericarp in the nucellar region, presumably along a suberized surface. Hyphal morphology in this region was variable with numerous swellings, in contrast with the thin, thread-like hyphae typical of the limited saprophytic growth found on the outside of the pericarp and surrounding host tissue. 

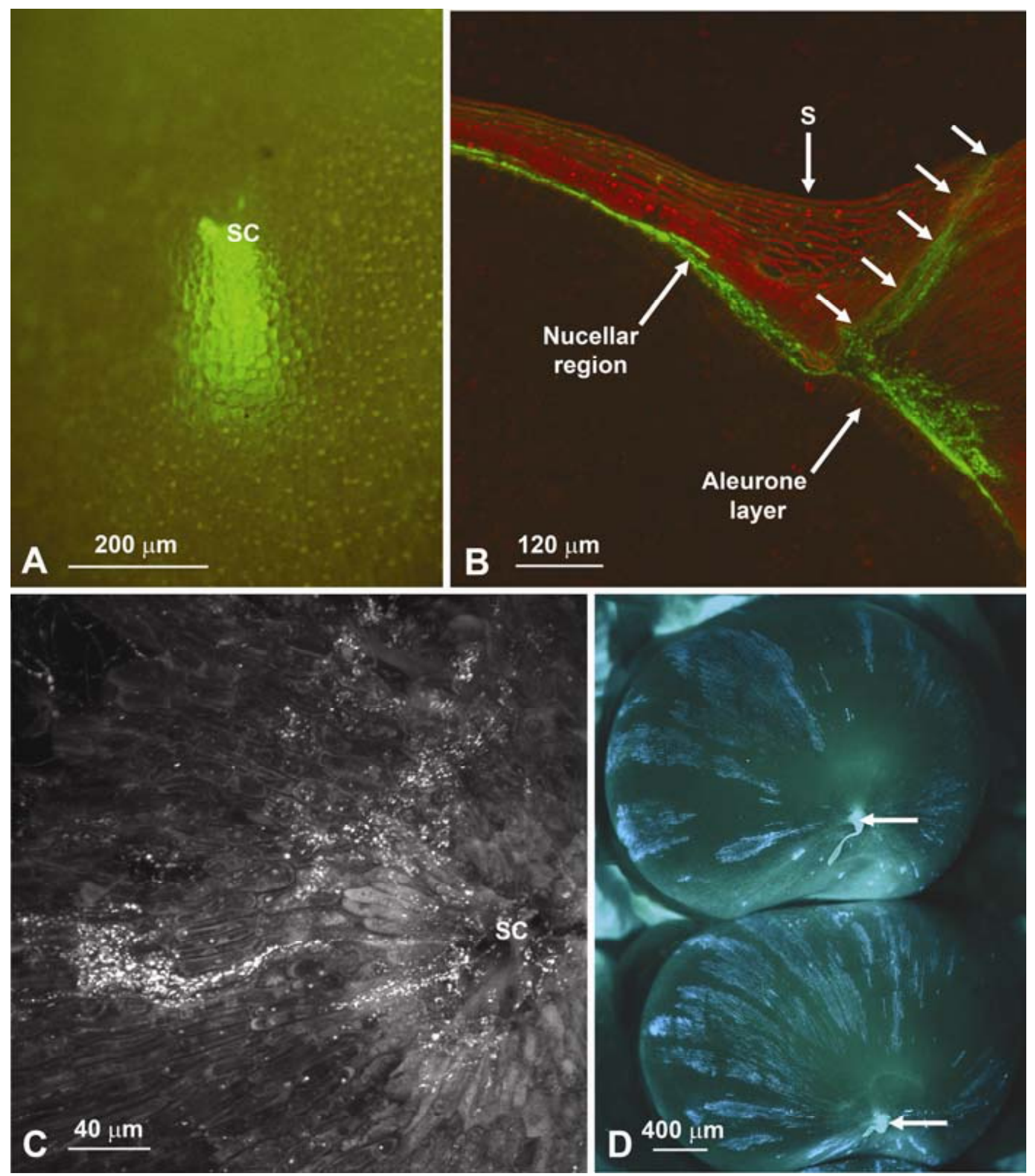

Fig. 5. ZsGreen-expressing Fusarium verticillioides colonization of AD38 maize kernels. A, Dissecting microscope image, acquired using a longpass emission filter, of fluorescent hyphae inside the stylar canal (SC) imaged through translucent cell layers of pericarp 14 days postinoculation (dpi). Autofluorescent host cell nuclei are visible. B, Multichannel confocal or multiphoton and $\mathbf{C}$, single-channel laser scanning microscope maximum intensity projections of $270.8-\mu \mathrm{m}$ and 45 0.7- $\mu \mathrm{m}$ optical sections, respectively. B, Vibratome section through the stylar canal and silk scar region 16 dpi. Hyphae (green) occupied the full length of the stylar canal (arrows) and appeared in a radial pattern from the stylar canal outward in the nucellar region just outside the aleurone layer. Autofluorescence of host tissues is evident (red). S = kernel surface. C, Underside of a peeled pericarp in the region around the stylar canal (SC), 14 dpi. ZsGreen was sequestered in subcellular compartments, visible as dots of bright fluorescence in this image, within older hyphae so as to impart a discontinuous appearance of the planar mycelium. Host cells radiated from the stylar canal and were visible due to faint autofluorescence. D, Distinctive pattern of colonization by $F$. verticillioides growing endophytically within the pericarp. Macroscopic symptoms were not evident at this stage. Silk scar (indicated by arrows) is brightly autofluorescent (bandpass emission filter). 
plete degeneration of the kernel. The infection process described above applied only to AD38 kernels; no colonization of HT1 was observed unless the pericarp was mechanically breached as described in the Methods section. Only then was the fungus able to colonize the HT1 kernel (Fig. 8). Furthermore, in experiments where the pericarp was removed, dipped in an aqueous suspension of $F$. verticillioides conidia, and incubated in a moist chamber, fungal colonization was equally extensive on the surface and within the excised pericarp tissue of both lines of maize.

\section{Reincubation of infected kernels.}

Environmental conditions played a significant role in the development of macroscopic symptomatology. We identified kernels with pericarp colonized by ZsGreen-expressing $F$. verticillioides but showing no macroscopic symptoms. This was typical of kernels infected as described above. Kernels with this type of endophytic infection were reincubated by placing kernels in a moist chamber for up to $72 \mathrm{~h}$. In this moist environment, the fungus rapidly grew up through the pericarp and sporulated readily on the kernel surface in conspicuous starburst patterns that were visible macroscopically (Fig. 9). We presumed that this reincubation mimicked the common practice used during disease evaluations in field trials of placing mature ears on the ground overnight before disease assessment.

\section{DISCUSSION}

The intact and uninjured pistillate inflorescence, or ear, of maize typically presents a formidable barrier to pathogens that infect specific tissues that lie under several to many overlapping leaf sheaths or husks. In the case of kernel rot fungal pathogens that infect kernel tissues, there is the additional bar-
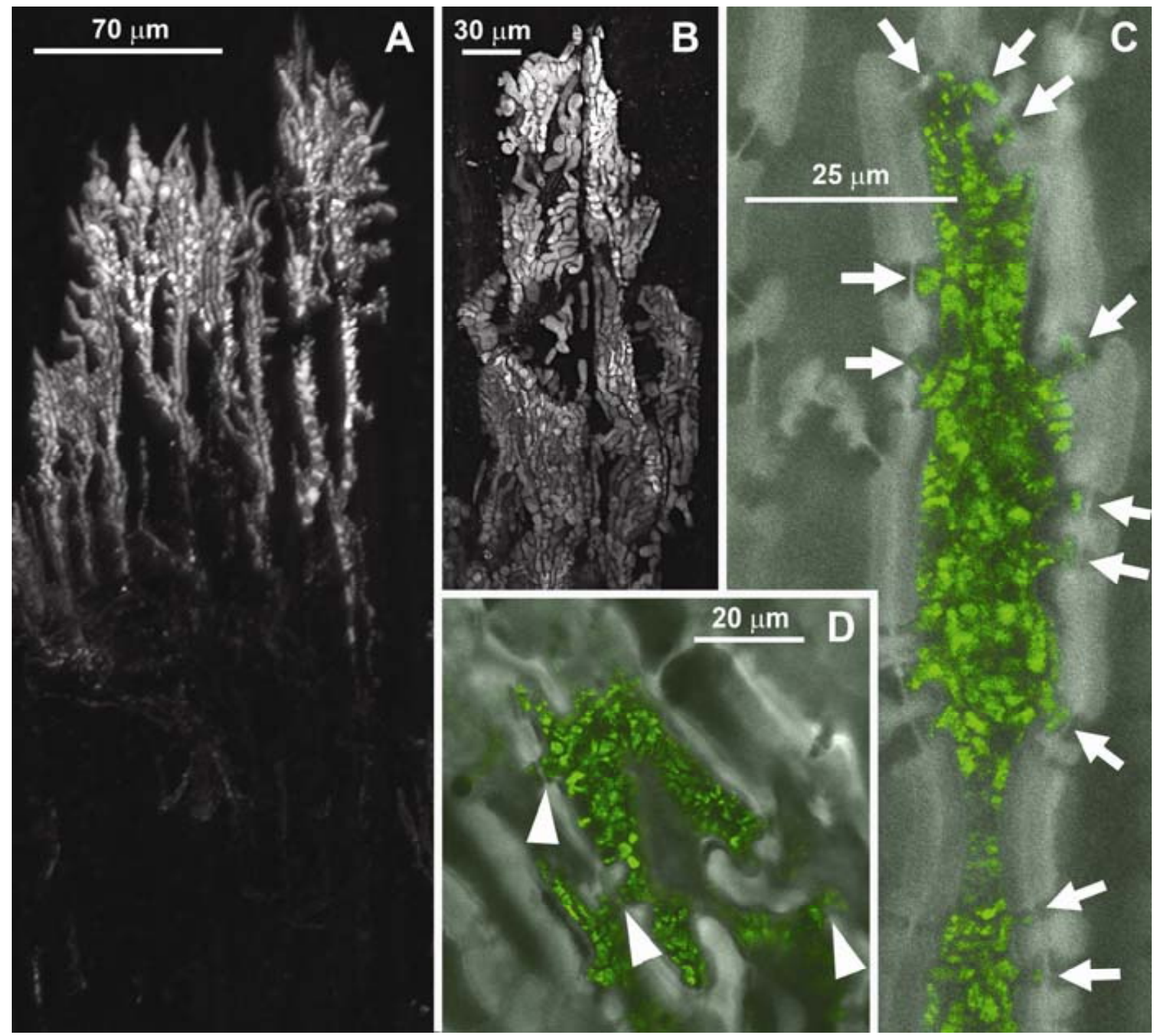

Fig. 6. Confocal laser scanning microscopy (LSM) images of ZsGreen-expressing Fusarium verticillioides hyphae colonizing the pericarp of AD38 kernels, rendered as maximum intensity projections of $\mathbf{A}, 421.25-\mu \mathrm{m}$ and $\mathbf{B}, 470.7-\mu \mathrm{m}$ optical sections. Hyphae within the long cells of the middle pericarp (mesocarp), 29 days postinoculation (dpi). Note the brighter hyphae at the actively growing margin of the mycelium (upper region of A compared with lower region). The characteristic lobed morphology of the fungal cells is particularly clear in B. C and D, Multichannel confocal or multiphoton LSM single optical sections $0.6 \mu \mathrm{m}$ thick of tissue fixed at $32 \mathrm{dpi}$. The pathogen has grown and fully occupied $\mathbf{C}$, a single file of pericarp cells or $\mathbf{D}$, cells of adjacent host cell files. Note the numerous pit pairs within this host tissue, and the positioning of hyphae against pit membranes (indicated by arrows, C). At this endophytic stage, hyphae spread to neighboring cells via penetration of pit membranes (indicated by arrowheads, D). 
rier of the intact pericarp. Together, these defensive structures usually protect the host fruit from what may be a constant and massive onslaught of inoculum. Because $F$. verticillioides, as well as the other kernel rot pathogens, cannot apparently form penetration structures such as appressoria that might otherwise allow the fungus to breach these barriers directly (Howard 1997) prior to extensive senescence of host tissues, there must be other avenues for ingress.

We have mentioned above the well-established role that injury of host tissues can play in facilitating $F$. verticillioides pathogenesis. The focus of the current study was on the possibility that this fungus and, perhaps, other kernel rot pathogens - in particular, those associated with the starburst symptom (Payne 1999) — enter kernels that are not injured, not fed upon by pests, and not cracked by nonoptimal conditions during maturation and drying. The possibility that these pathogens can defeat the barrier posed by the husks and enter ears through the silk channel has been shown previously, as discussed above. Koehler (1942) noted that only a few fungal taxa were often found associated with silks taken from under husks at mid-ear under field conditions and cautioned that these fungi should not be considered to have arrived there via "contamination." Rather, he inferred and implied fungal specialization that could serve as a component of pathogenesis. How these fungi entered ears, in the absence of vectors, might include i) the growth of hyphae along the outside of silks (stigmas) from outside the husk covering to inside; ii) penetration into the exposed silks that hang outside the husks and growth within stylar tissue, perhaps following the path of pollen tubes down to the kernel; or iii) movement of conidia from exposed silks to regions deep inside the husk-covering facilitated by movement of free water, perhaps dictated under forces of capillarity. Based on the fact that growth of some pathogens along the path of pollen tubes was already known (Shinners and Olson 1996; McManus et al. 1999; Ngugi and Scherm 2004), and because the starburst symptom appears, upon casual observation, to form from the silk-pericarp interface, we conducted preliminary studies specifically to test for that avenue of ingress. Using spray inoculation of pollinated and unpollinated ears, we found no evidence for either of the first two possibilities.

The primary focus of our subsequent efforts was on elucidating a possible path for pericarp ingress, taking as an assumption that the husk barrier could be breached under certain environmental conditions. Clearly, environmental conditions represent a highly significant factor; for example, as invoked to explain different experimental results when silks were spray inoculated in the greenhouse versus in the field (Reid et al. 1992). Undoubtedly, many factors-including fungal species and isolate, host genotype, and conditions of development, as well as environmental parameters during all stages of hostpathogen interaction-play important and almost certainly pivotal roles during pathogenesis (Maiorano et al. 2009). The complexity imposed on the pathosystem by so many possible variables, and the lack of sufficient controls, represent significant impediments to understanding the disease process.
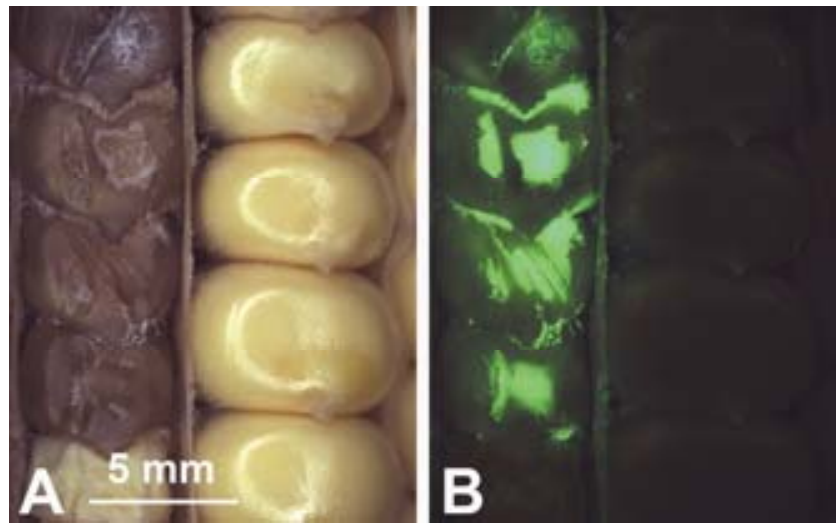

Fig. 8. Same region of an ear from resistant line HT1 (28 days postinoculation) and imaged using $\mathbf{A}$, visible light and $\mathbf{B}$, fluorescence. A, Kernel rot was evident only in those kernels that were mechanically damaged immediately prior to inoculation (kernels on the left). B, Damaged and rotted kernels exhibited massive colonization by ZsGreen-expressing Fusarium verticillioides, whereas the adjacent row of undamaged kernels did not.

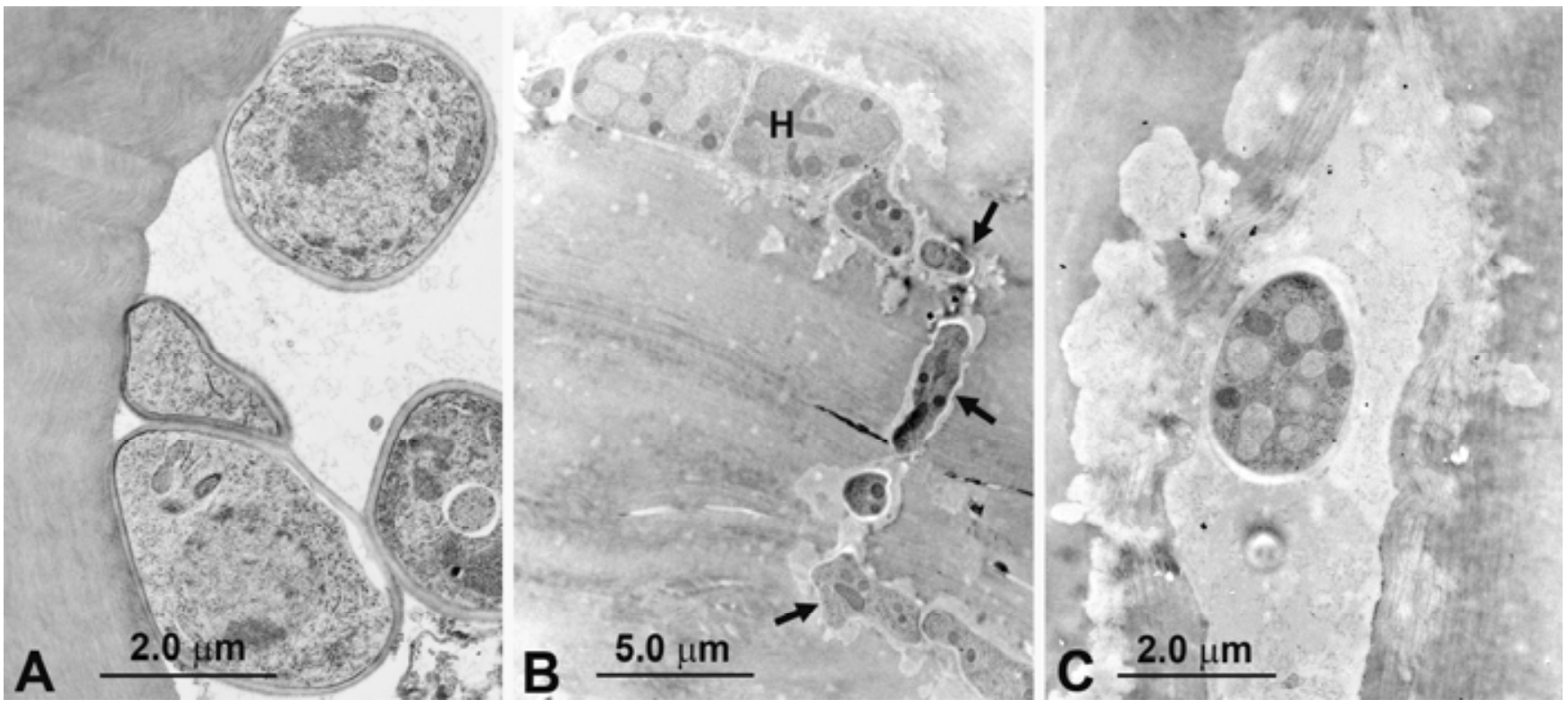

Fig. 7. Transmission electron microscope images of Fusarium verticillioides hyphae growing within the pericarp of a susceptible Gaspe flint line of maize. A, During endophytic growth stages, hyphae readily grew within the empty lumen of the long thick-walled cells of pericarp, without conspicuous damage to host cell walls. B and $\mathbf{C}$, Host cell wall dissolution by $F$. verticillioides was responsible for macroscopic symptoms that were manifest as white streaks in the pericarp. Note extensive dissolution of host cell wall juxtaposed with hyphae $(\mathrm{H})$. Narrower peg-like fungal cells (indicated by arrows) facilitated ramification directly through the thickened secondary walls of host cells. Specimens prepared by high-pressure freezing and freeze substitution. 
To simplify and allow us to better define pathogenesis, and to document the mode of ingress into kernels, we adopted the inoculation method of introducing a suspension of conidia into the silk channel under the controlled experimental conditions afforded by growth chambers. This approach led us to identify the stylar canal (Miller 1919) as a previously undocumented infection court. It should be noted that fungal infection by growth through the "stylar canal" has been reported (Lehman et al. 2007); however, the structure in question and use of the term were different than that reported here. In the Shinners and Olson study (1996), the pathogen grew through the pollen-transmitting tissue of the style (Heslop-Harrison et al. 1984), which they referred to as the stylar canal, as would Esau (1965) and Marsh and Payne (1984), for example. In the present study, the term is used as defined by Miller (1919) and Randolph (1936) — after Guignard (1901), who incorrectly believed it represented the
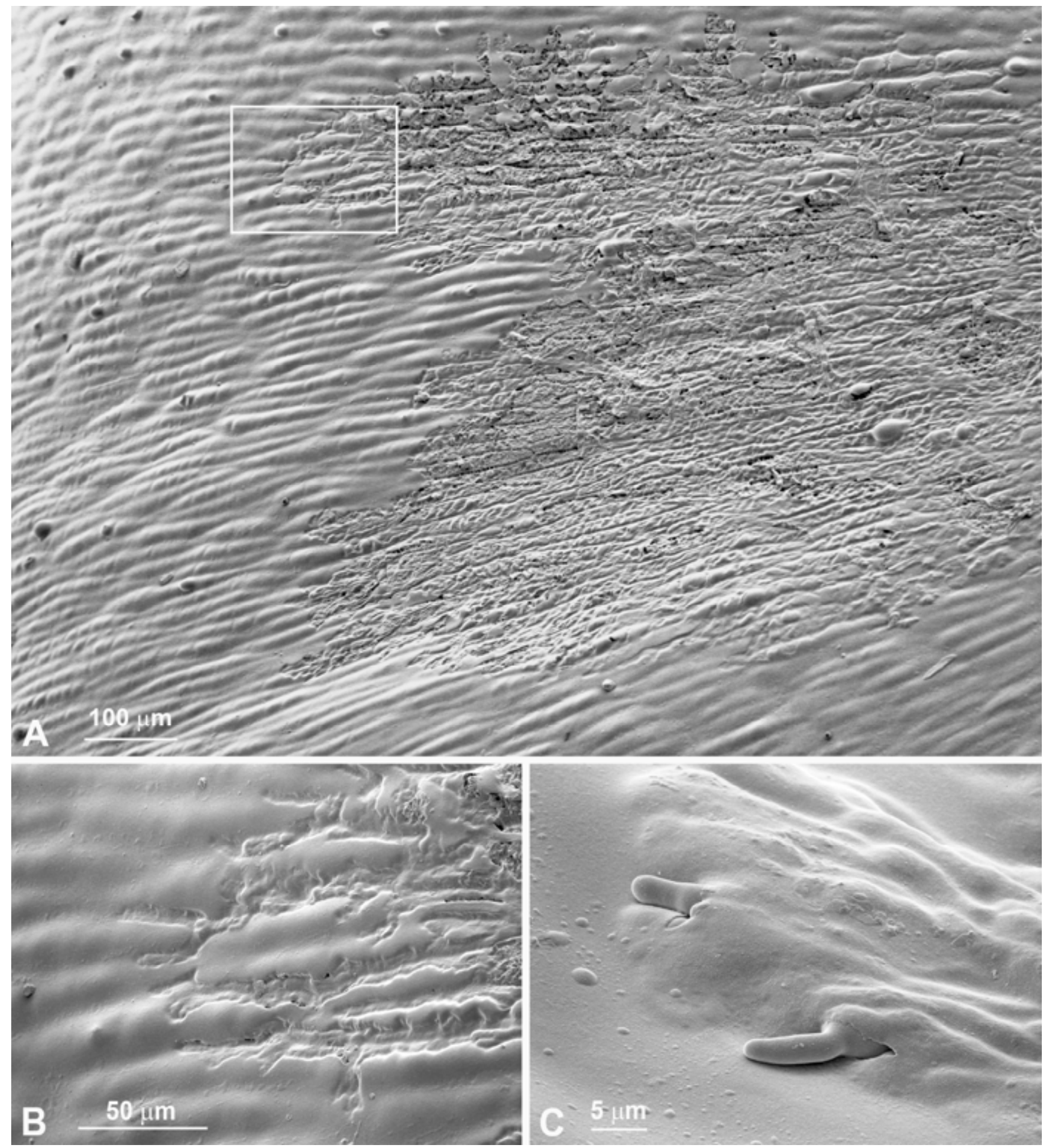

Fig. 9. Surface of AD38 kernels 21 days postinoculation, screened for endophytic ZsGreen-expressing Fusarium verticillioides colonization of the pericarp with a fluorescence dissecting microscope, and subsequently reincubated in a moist chamber for $72 \mathrm{~h}$. A, Collapse of the pericarp surface occurred in a pattern that matched that of colonization exactly. B, Enlarged region of A inset. C, Leading edge of one of the streaks in the pericarp. Note hyphae have erupted through to the pericarp surface-massive conidiation followed. Cryo scanning electron microscopy images. 
path to the ovule taken by pollen tubes, and named the structure accordingly. Thus, in maize, the term "stylar canal" has become known as the opening through the ovary wall (pericarp) formed where the two anterior and one posterior carpels join (Bonnett 1940; Kiesselbach 1949; Cheng et al. 1983; Heslop-Harrison et al. 1985; Stevens et al. 1986), and is unrelated to the pollen transmitting tissues referred to as "stylar canal" by other authors.

SEM observations provided no evidence for the presence of microscopic breaks in the pericarp that might allow the pathogen to enter. Indeed, fluorescence microscopy established that there was little fungal growth on the surfaces of kernels, and we never observed any signs of direct penetration into an intact pericarp. The surface of the pericarp has been reported to be covered by a thin layer of cutin (Johann 1935; Wolf et al. 1952; Heslop-Harrison et al. 1985) and, as such, might represent a barrier to penetration by some pathogens. It is also possible that the surface cuticle is continuous with and lines the walls of the stylar canal as well. Thus, the stylar canal would represent the only route to the pericarp cells from outside of the kernel and that, in the absence of injury, could be followed by pathogens without the need (or ability) to breach cuticles. We hypothesize that conidia move readily with free water through the silk channel and that hyphae grow on the cuticle surface, thereby entering the kernel through and to the bottom of the stylar canal, and then grow laterally in the various spaces that can be found between the "nucellar membrane" (Johann 1935; Randolph 1936) and inner pericarp epidermis-a space that is also occupied by integuments or remnants thereof. Apparently, several surfaces of tissues in this area-between the aleurone and inner pericarp epidermis - are suberized (Johann 1942; Heslop-Harrison 1985), some discontinuously so. From this extracellular infection court, hyphae enter the somewhat elongated epidermal cells of the inner pericarp surface and then of adjacent outer areas of the pericarp, which consist of files of cells with walls that thicken as they mature, becoming lignified and highly pitted (Johann 1935; Randolph 1936) and are arranged in a radial pattern "from a focus near the [bottom] exit of the stylar canal" (Heslop-Harrison et al. 1985). Hyphae fill the lumens of these cells quickly and grow more slowly from cell to cell, spreading through pits, forming the same radial pattern. Thus the starburst symptom is based upon the pattern of host cells that are colonized by the fungus.

There may be some correlation between the discontinuous areas of nucellus that lack suberized surfaces (as noted above) and instances where the pathogen enters and ramifies within the pericarp. This could be a reason for apparent discontinuities, or gaps, in the fluorescent streaks (Fig. 5D). Additionally, ZsGreen signal was brightest at the young margin of mycelia, indicating that age or condition of fungal cells may also be relevant factors.

Both fluorescence microscopy and SEM provided convincing evidence for entry through the stylar canal and alerted us to structural differences between the lines of maize used in this study. In fact, we infer from these observations that the stylar canal phenotype, being open or closed, was, under our experimental conditions, the sole reason for differential resistance and susceptibility. Experiments where the pericarp was mechanically wounded or removed and incubated with the fungus in vitro corroborate this hypothesis.

As reported here, the open versus closed appearances of the stylar canal in lines AD38 and HT1 were evident very early in development and remained so through maturity. On the other hand, Miller (1919) examined various lines of dent corn and found that the open stylar canals became closed by the time the silk was ready for pollination. Wolf and associates (1952) stated that "in spite of its name" the stylar canal of their specimen ker- nels was not open and they did not consider it as a possible avenue for pathogen ingress. To make inferences regarding possible pathogen ingress based upon an open or closed stylar canal in a particular maize line may be further complicated by variation in tissues underlying the pericarp as well. For example, Weatherwax (1917) observed that outer integument can become folded at the top of the ovary, forming a wedge of tissue "closing the stylar canal." In terms of the significance of stylar canal architecture relative to resistance to kernel rot diseases, there would appear to be numerous maternal genetic possibilities. This line of thinking deserves further investigation (Headrick and Pataky 1991; Sampietro et al. 2009), especially with recent advances in genomic analysis. For example, it may be useful to sort kernels of maize lines according to stylar canal architecture and then compare the groupings with kernel rot severity data observed under various conditions. This type of analysis might be used to establish a genetic basis for stylar canal phenotype and as a means to select maize lines for increased disease resistance. Regardless, any claims of positive correlation between these phenotypes and real utility would require a more sophisticated ability to measure levels of endophytic infection than is currently available under field conditions.

Koehler (1942) discussed instances where ears were inoculated at tips and later exhibited butt rot, and "the fungus apparently made its way down the ear without prominent visible signs." During a previous study of a different pathosystem, Septoria blotch of wheat (Duncan and Howard 2000), we were struck by seemingly opposing roles of extracellular matrices (ECM) associated with conidia at different stages of development. Traditional thinking has established a role for ECM in adhesion of conidia and germlings to various surfaces (Braun and Howard 1994). However, our observations of nearly instant and far-reaching dispersal of Mycosphaerella graminicola conidia on the surface of water upon contact with a mass of mucilage-embedded spores of a cirrus provided vivid demonstration of the power and efficiency of an apparently hydrophobic ECM-facilitated dispersal mechanism. With these ideas in mind, we speculate that conidia of kernel rot fungi have a related dispersal mechanism and that, under specific environmental circumstances, free water-and conidia-move through the silk channel, perhaps aided by forces of capillarity derived from highly disproportional surface-to-volume ratios: thus, tightly packed green silks would be expected to offer more movement of the fungus than would senescing silks. This idea would be consistent with reports of a curious direct relationship between lower disease incidence and older (senescent) silks (Sutton 1982; Reid et al. 1992, 2002): as silks collapse and become separated from one another in the silk channel, one might expect diminished opportunity for capillary forces. The copious production of conidia on exposed silks in association with pollen reported in the current study_noted also by Miller and associates (2007), with a similar observation by Naik and Busch (1978) for F. graminearum, and Marsh and Payne (1984) for Aspergillus flavus - could serve the pathogen well in such a scenario. Water would move conidia down the surface of silks to the base where, in the case of line AD38, conidia would be funneled by the trough juxtaposed with the gaping stylar canal and, thereby, gain direct access into the kernel interior.

\section{MATERIALS AND METHODS}

Corn lines (susceptible line AD38 and resistant line HT1) were provided by Pioneer, DuPont Agriculture \& Nutrition (Johnston, IA, U.S.A.). A susceptible Gaspe flint line was used for TEM analysis. Seedlings were grown on the greenhouse bench in 4-in. flats containing Fafard germinating mix 
(Conrad Fafard, Inc. Agawam, MA, U.S.A.). Seedlings were watered as needed and transplanted after 2 to 3 weeks into 9in. pots with Metro Mix 700 (Scotts, Marysville, OH, U.S.A.), fertilized with Osmocote 15-9-12 (Scotts) and Ironite 1-0-1 (Walnut Creek, CA) at $8 \mathrm{~g} / \mathrm{pot}$, and treated with Marathon $1 \% \mathrm{G}$ systemic insecticide (OHP, Mainland, PA, U.S.A.) at $5 \mathrm{~g} /$ pot. Insects were additionally controlled as needed with a rotating treatment regimen of Avid $0.15 \mathrm{EC}$ (Syngenta, Greensboro, NC, U.S.A.), DuraGuard ME (Whitmire Micro-Gen, St. Louis), Mavrik Aquaflow (Wellmark, Schaumburg, IL, U.S.A.), and Talstar GH (Whitmire MicroGen) according to published label rates. Auxiliary greenhouse lighting consisted of a combination of metal halide (400-W) and sodium vapor $(1,000-\mathrm{W})$ lamps producing approximately $400 \mu \mathrm{E}$ at bench-top level. Ears were covered with wax paper shoot bags just prior to silk emergence to allow for controlled pollinations. Plants were self pollinated whenever possible and sibling pollinated when necessary; AD38 and HT1 were never cross pollinated in this study. Freshly collected pollen was applied when silks had emerged a minimum of $2 \mathrm{~cm}$ from the top of the husk. Pollinated ears were covered with paper pollination bags to prevent further pollination. Only one ear per plant was pollinated. Shoot bags remained in place on unpollinated control ears until inoculation.

A culture of wild-type strain M-8114 was originally obtained by J. A. Sweigard (DuPont Crop Protection) from the Fusarium Research Center Culture Collection and confirmed as F. verticillioides by comparing the sequence of a region of $\beta$-tubulin to GenBank sequence submissions. F. verticillioides transformant strains Fvd32 and Fvd40 expressing ZsGreen reef coral fluorescent protein, a gift from James A. Sweigard, were used in this study. Methods for transformation, in vitro growth rate, and pathogenicity of ZsGreen-expressing individuals relative to the wild type were reported previously (Bourett et al. 2002).

Novel materials described in this publication may be available for noncommercial research purposes upon acceptance and signing of a material transfer agreement. In some cases, such materials may contain or be derived from materials obtained from a third party. In such cases, distribution of material will be subject to the requisite permission from any third-party owners, licensors, or controllers of all or parts of the material. Obtaining any permissions will be the sole responsibility of the requestor.

Upon initial receipt of $F$. verticillioides expressing ZsGreen, a transfer was made to quarter-strength potato dextrose agar plates, prepared by using BD Difco potato dextrose broth at 6 $\mathrm{g} /$ liter solidified with BD Bacto agar at $17 \mathrm{~g} / \mathrm{liter}$ (Becton Dickenson and Co., Sparks, MD, U.S.A.), and the fungus was incubated for 7 days at $22^{\circ} \mathrm{C}$. For long-term storage, conidia were stored in $50 \%$ glycerol at $-80^{\circ} \mathrm{C}$. Inoculum was prepared from frozen stocks. Ear inoculations were done with conidial suspensions of $1 \times 10^{6}$ conidia/ml of water as described below.

Maize plants remained in the greenhouse for 7 days after pollination, at which time they were moved to a growth chamber for inoculation. The growth chamber (Conviron, Winnipeg, Canada), with a growth height of $3.7 \mathrm{~m}$, was set to $26^{\circ} \mathrm{C}$ for 16 -h days, $20^{\circ} \mathrm{C}$ for 8 -h nights, $70 \%$ relative humidity for $24 \mathrm{~h}$, and provided lighting with metal halide bulbs $(1,000 \mathrm{~W})$ producing $600 \mu \mathrm{E}$ at $1 \mathrm{~m}$ from the floor. Ears were inoculated 24 $\mathrm{h}$ after plants were moved into the growth chamber. Pollination bags or shoot bags remained on the ears until immediately prior to inoculation. For spray inoculation, a hand-held Preval sprayer (Precision Valve Corp., Yonkers, NY, U.S.A.) was used to apply conidial suspension to exposed silks just to the point of run-off (approximately $4 \mathrm{ml}$ ). For injection inoculation, a syringe fitted with an 18-guage needle was used to introduce $10 \mathrm{ml}$ of the conidial suspension into the silk channel (Miller et al. 2007; Yates and Sparks 2008) and flood the entire devel- oping ear: an inoculation was deemed successful when none of the $10-\mathrm{ml}$ suspension leaked out. Inoculated ears were covered with loose-fitting, clear plastic bags for $48 \mathrm{~h}$, after which time the bags were removed (Headrick and Pataky 1991; Reid et al. 1992; Munkvold et al. 1997). A hand-held plant mister filled with sterile water was used to lightly mist the silks of inoculated ears once each morning until harvest to simulate dew on exposed silks. For injury experiments, a lead frog (weighted base with sharpened brass nails, used in flower arrangements) was used to puncture developing kernels by carefully striking the husk at a right angle to the axis of the ear, immediately prior to inoculation as above.

For light microscopy, ears were collected at 5 to $35 \mathrm{dpi}$. After removing husks, kernels of interest were selected using a Lumar model fluorescence stereomicroscope (Carl Zeiss MicroImaging Inc., Thornwood, NY, U.S.A.) with 450- to 490-nm bandpass excitation and either 500- to 550-nm BP or 505-nm longpass emission, detached from the ear, and fixed overnight in $2 \%$ paraformaldehyde in phosphate buffered saline (PBS) $(\mathrm{pH} 7.4)$ at $25^{\circ} \mathrm{C}$ (Sigma P3813), rinsed $2 \times$ in PBS, and stored at $4^{\circ} \mathrm{C}$. Sections, $125 \mu \mathrm{m}$ thick, were taken from whole kernels using a Pelco 100 Vibratome (Ted Pella, Inc, Redding, CA, U.S.A.). To examine excised pericarp tissue, fine forceps were used to carefully peel the pericarp from the kernel surface. Sections of peeled pericarp were mounted in water under a cover glass and the edges sealed with paraffin. Conventional fluorescence imaging was done on an Axiophot model fluorescence microscope (Carl Zeiss) with a $\times 20$ Plan-Neofluor $(0.5$ NA) objective lens, 450- to 490-nm bandpass excitation, a 510-nm beam splitter, and a 515-nm longpass emission filter. Images from both the Lumar and Axiophot were captured using a AxioCam HRc digital camera and AxioVision 4.4 image capture software (Carl Zeiss). For confocal and multiphoton imaging, a model LSM510 microscope (Carl Zeiss) was used with either a $\times 20$ Plan-Apochromat $(0.75 \mathrm{NA})$ or a $\times 40 \mathrm{C}$ Apochromat (1.2 NA water immersion) objective lens. Multichannel imaging of ZsGreen was as described previously (Bourett et al. 2002). In some samples (not shown), pericarp autofluorescence was enhanced by fixation in glutaraldehyde and then imaged with 720-nm two-photon excitation provided by a Chameleon tunable laser (coherent) and 685 longpass emission. Image capture and analysis used AIM software (version 4.0 SP2; Carl Zeiss).

Samples for cryoSEM were prepared and examined as described previously (Braun and Howard 1994). Pericarp tissue for high pressure freezing (HPF) and TEM was taken at $21 \mathrm{dpi}$ from infected Gaspe flint kernels. Pericarp from infected kernels was peeled as described above and examined with a fluorescence stereomicroscope, and regions of the pericarp containing ZsGreen-expressing hyphae were identified. A 1.2-mm tissue biopsy punch was used to collect samples of infected pericarp. Tissue punches were immediately frozen in 1-hexadecene (Sigma-Aldrich, St. Louis) using an EMPACT high pressure freezer (Leica Microsystems, Bannockburn, IL, U.S.A.). High-pressure frozen samples were substituted in $4 \%$ $\mathrm{OsO}_{4}$ in anhydrous acetone at $-80^{\circ} \mathrm{C}$ for $72 \mathrm{~h}$. Samples were then transferred to an automated freeze substitution apparatus (Leica Microsystems) programmed to carry out the following warm-up schedule: $-85^{\circ} \mathrm{C}$ for $30 \mathrm{~min}$, ramped from -85 to $30^{\circ} \mathrm{C}$ at $5^{\circ} \mathrm{C} / \mathrm{h}$, held at $-30^{\circ} \mathrm{C}$ for $7 \mathrm{~h}$, ramped from -30 to $0^{\circ} \mathrm{C}$ at $5^{\circ} \mathrm{C} / \mathrm{h}$, and held at $0^{\circ} \mathrm{C}$ for $1 \mathrm{~h}$. Samples were then transferred to room temperature for $90 \mathrm{~min}$ before rinsing in anhydrous acetone containing 1\% acidified 2,2-dimethyoxypropane (Electron Microscopy Sciences, Hatfield, PA, U.S.A.). Samples were infiltrated gradually in an ascending graded series of Epon resin (Studer et al. 1995) in acetone before transferring to rubber molds with fresh resin and polymerizing at $60^{\circ} \mathrm{C}$ for 
$96 \mathrm{~h}$. Ultrathin sections were stained for $10 \mathrm{~min}$ with $2 \%$ aqueous uranyl acetate followed by $5 \mathrm{~min}$ in Reynold's lead citrate and examined with a model EM420 transmission electron microscope (FEI, Hillsboro, OR, U.S.A.) operated at $100 \mathrm{kV}$.

\section{ACKNOWLEDGMENTS}

We thank J. A. Sweigard (DuPont Crop Protection) for a culture of the transgenic ZsGreen-expressing F. verticillioides and T. M. Bourett (DuPont $\mathrm{Ag}$ Biotech) for sample preparation and transmission electron microscopy.

\section{LITERATURE CITED}

Bacon, C. W., Glenn, A. E., and Yates, I. E. 2008. Fusarium verticillioides: managing the endophytic association with maize for reduced fumonisins accumulation. Toxin Rev. 27:411-446.

Bonnett, O. T. 1940. Development of the staminate and pistillate inflorescences of sweet corn. J. Agric. Res. 60:25-37.

Bourett, T. M., Sweigard, J. A., Czymmek, K. J., Carroll, A., and Howard, R. J. 2002. Reef coral fluorescent proteins for visualizing fungal pathogens. Fungal Genet. Biol. 37:211-220.

Braun, E. J., and Howard, R. J. 1994. Adhesion of fungal spores and germlings to host surfaces. Protoplasma 181:202-212.

Cheng, P. C., Greyson, R. I., and Walden, D. B. 1983. Organ initiation and the development of unisexual flowers in the tassel and ear of Zea mays. Am. J. Bot. 70:450-462.

Dowd, P. F. 1998. Involvement of arthropods in the establishment of mycotoxigenic fungi under field conditions. Pages 307-350 in: Mycotoxins in Agriculture and Food Safety. K. K. Sinhaand and D. Bhatagnar, eds. Marcel Dekker, New York.

Duncan, K. E., and Howard, R. J. 2000. Cytological analysis of wheat infection by the leaf blotch pathogen, Mycosphaerella graminicola. Mycol. Res. 104:1074-1082.

Esau, K. 1965. Plant Anatomy, 2nd ed. Wiley \& Sons. New York.

Guignard, L. 1901. La double fecundation dans le mais. J. Bot. 15:37-50.

Headrick, J. M., and Pataky, J. K. 1991. Maternal influence on the resistance of sweet corn lines to kernel infection by Fusarium moniliforme. Phytopathology 81:268-274.

Headrick, J. M., Pataky, J. K., and Juvik, J. A. 1990. Relationships among carbohydrate content of kernels, condition of silks after pollination, and the response of sweet corn inbred lines to infection of kernels by Fusarium moniliforme. Phytopathology 80:487-494.

Heslop-Harrison, Y., Reger, B. J., and Heslop-Harrison, J. 1984. The pollen-stigma interaction in the grasses. 6. The stigma ('silk') of Zea mays L. as host to the pollens of Sorghum bicolor (L.) Moench and Pennistem americanum (L.) Leeke. Acta Bot. Neerl. 33:205-227.

Heslop-Harrison, Y., Heslop-Harrison, J., and Reger, B. J. 1985. The pollen-stigma interaction in the grasses. 7. Pollen-tube guidance and the regulation of tube number in Zea mays L. Acta Bot. Neerl. 34:193-211.

Hesseltine, C. W., and Bothast, R. J. 1977. Mold development in ears of corn from tasseling to harvest. Mycologia 69:328-340.

Howard, R. J. 1997. Breaching the outer barriers-cuticle and cell wall penetration. Pages 43-60 in: The Mycota V, Part A. Plant Relationships. G. C. Carroll and P. Tudzynski, eds. Springer-Verlag, Berlin.

Johann, H. 1935. Histology of the caryopsis of yellow dent corn, with reference to resistance and susceptibility to kernel rots. J. Agric. Res $51: 855-883$

Johann, H. 1942. Origin of the suberized semipermeable membrane in the caryopsis of maize. J. Agric. Res. 64:275-282.

Jones, R. K., Duncan, H. E., Payne, G. A., and Leonard, K. J. 1980. Factors influencing infection by Aspergillus flavus in silk-inoculated corn. Plant Dis. 64:859-863.

Kiesselbach, T. A. 1949. The structure and reproduction of corn. Res. Bull. 161. Univ. Nebr. Coll. Agric. Agric. Exp. Stn. Lincoln, NE, U.S.A.. (Republished 1999. Cold Spring Harbor Laboratory Press, Cold Spring Harbor, NY, U.S. A.)

Koehler, B. 1942. Natural mode of entrance of fungi into corn ears and some symptoms that indicate infection. J. Agric. Res. 64:421-442.

Lehman, J. S., Igarashi, S., and Oudemans, P. V. 2007. Host resistance to Monilinia vaccinii-corymbosi in flowers and fruits of highbush blueberry. Plant Dis. 91:852-856.

Maiorano, A., Reyneri, A., Sacco, D., Magni, A., and Ramponi, C. 2009. A dynamic risk assessment model (FUMAgrain) of fumonisin synthesis by Fusarium verticillioides in maize grain in Italy. Crop Prot. 28:243-256.

Marsh, S. F., and Payne, G. A. 1984. Scanning EM studies on the colonization of dent corn by Aspergillus flavus. Phytopathology 74:557-561.

McManus, P. S., Best, V. M., and Voland, R. P. 1999. Infection of cran- berry flowers by Monilinia oxycocci and evaluation of cultivars for resistance to cottonball. Phytopathology 89:1127-1130.

Miller, E. C. 1919. Development of the pistillate spikelet and fertilization in Zea mays L. J. Agric. Res. 18:255-266.

Miller, S. S., Reid, L. M., and Harris, L. J. 2007. Colonization of maize silks by Fusarium graminearum, the causative organism of Gibberella ear rot. Can. J. Bot. 85:369-376.

Munkvold, G. P. 2003. Cultural and genetic approaches to managing mycotoxins in maize. Annu. Rev. Phytopathol. 41:99-116.

Munkvold, G. P., and Hellmich, R. L. 2000. Genetically modified, insect resistant maize: implications for management of ear and stalk diseases. Plant Health Prog. doi:10.1094/PHP-2000-0912-01-RV. Published online.

Munkvold, G. P., McGee, D. C., and Carlton, W. M. 1997. Importance of different pathways for maize kernel infection by Fusarium moniliforme. Phytopathology 87:209-217.

Naik, D. M., and Busch, L. V. 1978. Stimulation of Fusarium graminearum by maize pollen. Can. J. Bot. 56:1113-1117.

Ngugi, H. K., and Scherm, H. 2004. Pollen mimicry during infection of blueberry flowers by conidia of Monilinia vaccinii-corymbosi. Physiol. Mol. Plant Pathol. 64:113-123.

Nirenberg, H. I., and O'Donnell, K. 1998. New Fusarium species and combinations within the Gibberella fujikuroi species complex. Mycologia 90:434-458.

Payne, G. A. 1999. Ear and kernel rots. Pages 44-47 in: Compendium of Corn Diseases, 3rd ed. D. G. White, ed. American Phytopathological Society Press, St. Paul, MN, U.S.A.

Randolph, L. F. 1936. Developmental morphology of the caryopsis in maize. J. Agric. Res. 53:881-916.

Reid, L. M., Bolton, A. T., Hamilton, R. I., Woldemariam, T., and Mather, D. E. 1992. Effect of silk age on resistance of maize to Fusarium graminearum. Can. J. Plant Pathol. 14:293-298.

Reid, L. M., Woldemariam, T., Zhu, X., Stewart, D. W., and Schaafsma, A. W. 2002. Effect of inoculation time and point of entry on disease severity in Fusarium graminearum, Fusarium verticillioides, or Fusarium subglutinans inoculated maize ears. Can. J. Plant Pathol. 24:162-167.

Rossi, V., Scandolara, A., and Battilani, P. 2009. Effect of environmental conditions on spore production by Fusarium verticillioides, the causal agent of maize ear rot. Eur. J. Plant Pathol. 123:159-169.

Sampietro, D. A., Vattuone, M. A., Presello, D. A., Fauguel, C. M., and Catalán, C. A. N. 2009. The pericarp and its surface wax layer in maize kernels as resistance factors to fumonisin accumulation by Fusarium verticillioides. Crop Prot. 28:196-200.

Shinners, T. C., and Olson, A. R. 1996. The gynoecial infection pathway of Monilinia vaccinii-corymbosi in lowbush blueberry (Vaccinium angustifolium). Can. J. Plant Sci. 76:493-497.

Sobek, E. A., and Munkvold, G. P. 1999. European corn borer (Lepidoptera: Pyralidae) larvae as vectors of Fusarium moniliforme, causing kernel rot and symptomless infection of maize kernels. J. Econ. Entomol. 92:503-509.

Stevens, S. J., Stevens, E. J., Lee, K. W., Flowerday, A. D., and Gardner, C. O. 1986. Organogenesis of the staminate and pistillate inflorescences of pop and dent corns: relationship to leaf stages. Crop Sci. 26:712-718.

Stewart, D. W., Reid, L. M., Nicol, R. W., and Schaafsma, A. W. 2002. A mathematical simulation of growth of Fusarium in maize ears after artificial inoculation. Phytopathology 92:534-541.

Studer, D., Michel, M., Wohlwend, M., Hunziker, E. B., and Buschmann, M. D. 1995. Vitrification of articular cartilage by high-pressure freezing. J. Microsc. (Oxf.) 179:321-332.

Sutton, J. C. 1982. Epidemiology of wheat head blight and maize ear rot caused by Fusarium graminearum. Can. J. Plant Pathol. 4:195-209.

Valdivia, E. R., Cosgrove, D. J., and Stephenson, A. G. 2006. Role of accelerated style senescence in pathogen defense. Am. J. Bot. 93:17251729

Vigier, B., Reid, L. M., Seifert, K. A., Stewart, D. W., and Hamilton, R. I. 1997. Distribution and prediction of Fusarium species associated with maize ear rot in Ontario. Can. J. Plant Pathol. 19:60-65.

Warfield, C. Y., and Davis, R. M. 1996. Importance of the husk covering on the susceptibility of corn hybrids to Fusarium ear rot. Plant Dis. 80:208-210.

Weatherwax, P. 1917. The development of the spikelets of Zea mays. Bull. Torrey Bot. Club 44:483-496.

Wilke, A. L., Bronson, C. R., Tomas, A., and Munkvold, G. P. 2007. Seed transmission of Fusarium verticillioides in maize plants grown under three different temperature regimes. Plant Dis. 91:1109-1115.

Wolf, M. J., Buzan, C. L., MacMasters, M. M., and Rist, C. E. 1952. Structure of the mature corn kernel. II. Microscopic structure of pericarp, seed coat, and hilar layer of dent corn. Cereal Chem. 29:334348.

Yates, I. E., and Sparks, D. 2008. Fusarium verticillioides dissemination among maize ears of field-grown plants. Crop Prot. 27:606-613. 